\title{
The Araucaria Project: the Baade-Wesselink projection factor of pulsating stars
}

\author{
Nicolas Nardetto ${ }^{1}$, Jesper Storm ${ }^{2}$, Wolfgang Gieren ${ }^{3}$, \\ Grzegorz Pietrzyński ${ }^{4}$, and Ennio Poretti ${ }^{5}$ \\ ${ }^{1}$ Laboratoire Lagrange, UMR7293, Université de Nice Sophia-Antipolis, CNRS, Observatoire \\ de la Côte d'Azur, Nice, France \\ email: Nicolas. Nardetto@oca.eu \\ ${ }^{2}$ Leibniz Institute for Astrophysics, An der Sternwarte 16, 14482, Potsdam, Germany \\ email: jstorm@aip.de \\ ${ }^{3}$ Departamento de Astronomía, Universidad de Concepción, Casilla 160-C, Concepción, Chile \\ email: wgieren@astro-udec.cl \\ ${ }^{4}$ Warsaw University Observatory, Al. Ujazdowskie 4, 00-478, Warsaw, Poland \\ email: pietrzyn@astrouw.edu.pl \\ ${ }^{5}$ INAF - Osservatorio Astronomico di Brera, Via E. Bianchi 46, 23807 Merate (LC), Italy \\ email: ennio.poretti@brera.inaf.it
}

\begin{abstract}
The projection factor used in the Baade-Wesselink method of determining the distance of Cepheids makes the link between stellar physics and the cosmological distance scale. A coherent picture of this physical quantity is now provided based on several approaches. We present the latest news on the expected projection factor for different kinds of pulsating stars in the Hertzsprung-Russell diagram.
\end{abstract}

Keywords. stars: oscillations (including pulsations), stars: atmospheres

\section{Short review on the projection factor of Cepheids}

For many decades the Cepheid stars have been used to calibrate the distance scale and the Hubble constant through their well-known period-luminosity (PL) relation (see Riess et al. 2011 and Freedman \& Madore 2010 for a review). Recently, using the BaadeWesselink (BW) method to determine distances of Cepheids, Storm et al. (2011a) found that the $K$-band PL relation is nearly universal and can be applied to any host galaxy largely independent of metallicity. The projection factor is a key quantity of the BW methods: it is used to convert the radial velocity variation into the pulsation velocity of the star. There are several ways to study the projection factor. One can use geometrical or static models, hydrodynamical analysis, or even direct observations when the distance of the star is known. In the purely geometric approach, two effects are considered only: the limb-darkening of the star (in the continuum) and the expansion of the atmosphere (at constant velocity). The projection factor is then an integration of the pulsation velocity field (associated with the line-forming region) projected on the line of sight and weighted by the surface brightness of the star, which is defined for instance by $I(\cos \theta)=1-u_{V}+$ $u_{V} \cos \theta$, where $u_{V}$ is the limb darkening of the star in the $V$ band and $\theta$ is the angle between the normal of the star and the line of sight (Claret et al. 2011). In this case, the geometric projection factor can be derived as follows: $p_{0}=3 / 2-u_{V} / 6$ (Getting 1934). However, this definition of the projection factor implies a specific method of the radial velocity determination, which is the first moment or centroid method (Burki et al. 1982). Depending on the limb-darkening considered for the Cepheid studied, the value of the projection factor is different: $p=24 / 17=1.415\left(u_{V}=0.60\right.$, Getting 1934), $p=1.375$ 
$\left(u_{V}=0.75\right.$, Van Hoof \& Deurinck 1952) or $p=1.360\left(u_{\mathrm{V}}=0.80\right.$, Burki et al. 1982). The latter value has been widely used in spectroscopy. Recently, Neilson et al. (2012) derived the geometrical projection factor as a function of the period and for several photometric bands using a radiative transfer in spherical geometry and found a slightly lower value of $p=1.33$ for $\delta$ Cep. Additional studies should be also mentioned like Gray \& Stevenson (2007) and Hadrava et al. (2009) in which a geometrical model is directly fitted to the observed spectral line profile (the pulsation velocity is then an output). Such approaches are formally consistent with the geometrical method.

The second approach to study the Baade-Wesselink projection factor is to consider a hydrodynamical model, which describes the dynamical structure of the atmosphere of the star (in particular the atmospheric velocity gradient). Using a so-called piston model in which the radial velocity curve is used as an input, Sabbey et al. (1995) found a mean value of the projection factor of $p=1.34$. However, this value was derived using the bi-sector method of the radial velocity determination (applied to theoretical line profiles), which unfortunately makes the comparison with other studies quite uncertain. On the other hand, using a self-consistent model of the pulsation (requiring few fundamental parameters such as the stellar mass, the luminosity, the effective temperature and the chemical composition), Nardetto et al. (2004) found that the atmospheric velocity gradient (and other dynamical effects) reduce the geometric projection factor (found at $p_{0}=1.39$ with the model) by about $9 \%$, leading to a projection factor of $p=1.27 \pm 0.01$. This value is however consistent with the Gaussian fit method of the radial velocity determination (applied to a spectral line with a typical depth of $D=0.2$ ). In this $9 \%$ decrease, $5 \%$ comes from the dynamical structure of the atmosphere and $4 \%$ from using the Gaussian fit method. Indeed later, Nardetto et al. (2007) provided a revised value of the projection factor, $p=1.33 \pm 0.02$, applicable together with the first moment method (and consistent with a plane parallel model atmosphere). It is worth noticing that the projection factor is generally supposed as being constant over the pulsation phase following Nardetto et al. (2006b).

If one uses the cross-correlated radial velocity (which includes many lines and also a Gaussian fit of the cross-correlated mean line profile with a typical depth of $D=0.25$ ), a lower value of the projection factor is found (by about $11 \%$ compared to the initial geometrical projection factor $p_{0}=1.39$ ), i.e. $p=1.25 \pm 0.05$ (Nardetto et al. 2009). One can say approximatively that in these $11 \%, 7 \%$ comes from the dynamical structure of the atmosphere and $4 \%$ from the Gaussian fit.

Mérand et al. (2005) applied the inverse Baade-Wesselink method using an infrared FLUOR/CHARA interferometric observation of $\delta$ Cep. In this approach, the projection factor is fitted, while the distance of $\delta$ Cep is known (from the Hubble Space Telescope (HST) parallax) at the $4 \%$ level (Benedict et al. 2007). They found $p=1.27 \pm 0.05$ (using the cross-correlation method to derive the radial velocity). Then, deriving the infrared surface brightness angular diameters of $\delta$ Cep, and applying again the inverse BW method, Groenewegen (2007) and Laney \& Joner (2009) found similarly a value of the projection factor of $p=1.27$. Later, Storm et al. (2011b) constrained directly the period-projection factor $(P p)$ relation using spectroscopic and photometric observations of Cepheids in the Large Magellanic Cloud (hereafter LMC). In this method, the zeropoint of the $P p$ relation is again based on the $H S T$ trigonometric parallaxes of Galactic Cepheids, but the slope is derived from the BW distances of LMC Cepheids (all Cepheids in the LMC used by Storm can be assumed to be at the same distance, leading to an extra constraint on the period projection factor relation). The corresponding value for $\delta$ Cep itself is $p=1.41 \pm 0.05$. It has been shown that the metallicity has no impact (at least theoretically) on the projection factor (Nardetto et al. 2011). Using a similar 


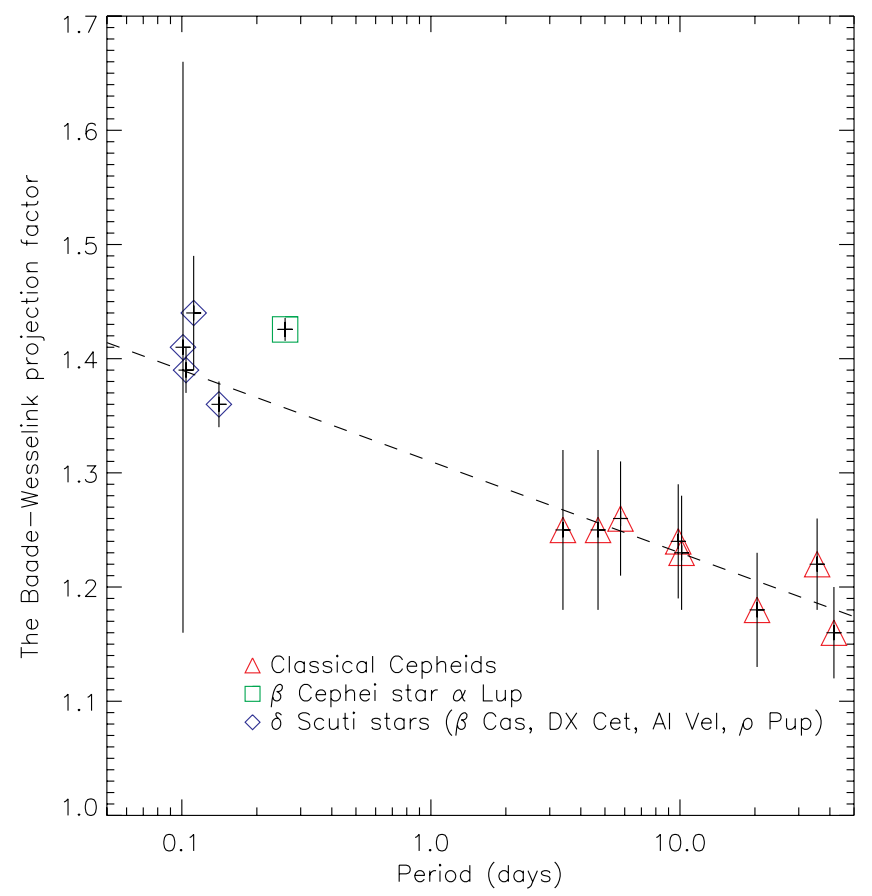

Figure 1. The Baade-Wesselink projection factor as a function of the period for different kinds of pulsating stars. The case of the $\beta$ Cephei star $\alpha$ Lup is described by Nardetto et al. (2013b).

method, Groenewegen (2013) found recently a value of the projection factor which is also quite high $(p=1.33)$. The latest result comes from Pilecki et al. (2013), who constrained the projection factor using a short-period Cepheid $(P=3.8$ days $)$ in an eclipsing binary system. They found $p=1.21 \pm 0.04$.

This short review shows that a lot of work has been done to constrain the BW projection factor. And even if some discrepancies remain concerning the inverse photometric BW method of determining the projection factor, a consensus is currently emerging. In particular, we emphasise that the fact that the projection factor derived from the surfacebrightness techniques is overestimated has no impact on the distances, because at the same time, the amplitude of the photometric angular diameter curve is underestimated. One can say finally that the photometric version of the BW method is self-consistently calibrated using the HST parallaxes to set the zero point and the distances to LMC Cepheids with a large range of periods to constrain the p-factor relation with pulsation period. However, Ngeow et al. (2012) found indeed a significant dispersion in the period-projection factor relation, and this should be also investigated.

\section{The projection factor for other types of pulsating stars}

One possible way to better understand the dynamical structure of Cepheids, and in particular the $k$-term $\dagger$ (Nardetto et al. 2006a, 2008), the mass loss (Nardetto et al. 2008), and the projection factor, is to perform comparison with other kinds of pulsating stars (as soon as they pulsate in a dominant radial model).

$\dagger$ The $k$-term is a residual asymmetry (not related to the pulsation) observed in the spectral line profiles of Cepheids. 
In the framework of the Araucaria Project (Gieren et al. 2005) of distances determination in the Local Group, we determined the Baade-Wesselink projection factor for four $\delta$ Sct stars: $\rho$ Pup $(p=1.36 \pm 0.02)$, DX Cet $(p=1.39 \pm 0.02)$, AI Vel $(p=1.44 \pm 0.05)$, and $\beta$ Cas $(p=1.41 \pm 0.25)$. (Refer to Nardetto et al. (2013a) for $\rho$ Pup and DX Cet and to Guiglion et al. (2013) for AI Vel and $\beta$ Cas.) Figure 1 shows how all these values fit in an excellent way the extension toward short periods of the relation found for Cepheids, i.e., $p=[-0.08 \pm 0.05] \log P+[1.31 \pm 0.06]$ (Nardetto et al. 2009). This result seems more robust than the similar one obtained by Laney \& Joner (2009) using an indirect method based on the comparison of geometric and pulsation parallaxes. On the other hand, the projection factor of the $\beta$ Cep star $\alpha$ Lup is $8 \sigma$ above the relation (Fig. 1). By omitting $\alpha$ Lup we can find a relation common to $\delta$ Sct stars and classical Cepheids.

\section{Conclusion}

The projection factor is a very complex quantity which involves all the physical structure of a Cepheid's atmosphere. Nevertheless, it is now well constrained using geometrical, hydrodynamical modelling and also direct observations (trigonometric parallaxes and interferometry). Thanks to these efforts to better understand the projection factor, the $\mathrm{BW}$ technique of distance determination is becoming one of the more robust methods in the path to the Hubble constant.

\section{References}

Benedict, G. F., McArthur, B. E., Feast, M. W., et al. 2007, AJ, 133, 1810

Burki, G., Mayor, M., \& Benz, W. 1982, A\&A, 109, 258

Claret, A. \& Bloemen, S. 2011, A\& $A$, 529, A75

Freedman, W. L. \& Madore, B. F. 2010, ARAA, 48, 673

Getting, I. A. 1934, MNRAS, 95, 139

Gieren, W., Pietrzyński, G., Bresolin, F., et al. 2005, The Messenger, 121, 23

Gray, D. F. \& Stevenson, K. B. 2007, PASP, 119, 398

Groenewegen, M. A. T. 2007, $A \mathscr{E} A, 474,975$

Groenewegen, M. A. T. 2013, A\& $A$, 550, A70

Guiglion, G., Nardetto, N., Mathias, P., et al. 2013, A\&A A, 550, L10

Hadrava, P., Šlechta, M., \& Škoda, P. 2009, A\&A, 507, 397

Laney, C. D. \& Joner, M. D. 2009, AIP-CP, 1170, 93

Mérand A., Kervella, P. \& Coudé du Foresto, V. 2005, A\& A, 447, 783

Nardetto, N., Fokin, A., Mourard, D., et al. 2004, A\&A, 428, 131

Nardetto, N., Mourard, D., Kervella, P., et al. 2006a, A\&3A, 453, 309

Nardetto, N., Fokin, A., Mourard, D., et al. 2006b, A\&A A, 454, 327

Nardetto, N., Mourard, D., Mathias, P. et al. 2007, A\&A, 471, 661

Nardetto, N., Stoekl, A., Bersier, D., et al. 2008a, A\&AA, 489, 1255

Nardetto, N., Groh, J. H., Kraus, S., et al. 2008b, A\&AA, 489, 1263

Nardetto, N., Gieren, W., Kervella P. et al. 2009, A\&AA, 502, 951

Nardetto, N., Fokin, A., Fouqué, P. et al. 2011, A\&A, 534, L16

Nardetto, N., Poretti E., Rainer M., et al. 2013a, AEGA, submitted

Nardetto, N., Mathias, P., Fokin, A., et al. 2013b, A\&A, 553, A112

Neilson, H. R., Nardetto, N., Ngeow, C.-C., et al. A\&SA, 541, A134

Ngeow, C.-C., Neilson, H. R., Nardetto, N., et al. 2012, A\&A, 543, A55

Pilecki, D., Graczyk, D., Pietrzyński, G., et al. 2013, MNRAS, 436, 953

Riess, A. G., Lucas M., Casertano S., et al. 2011, ApJ, 730, 119

Sabbey, C. N., Sasselov, D. D., Fieldus, M. S., et al. 1995, ApJ, 446, 250

Storm, J., Gieren, W., \& Fouqué, P. 2011a, AËA, 534, A94

Storm, J., Gieren, W., \& Fouqué, P. 2011b, A\&A, 534, A95

Van Hoof, A. \& Deurinck, R. 1952, ApJ, 115, 166 